\title{
Distribución de fuentes de ruido sísmico y una aplicación a la geofísica
}

\author{
Distribution of seismic noise sources and an application \\ to geophysics
}

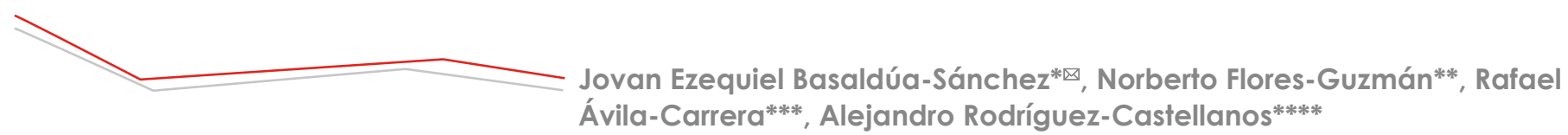

Basaldúa-Sánchez, J. E., Flores-Guzmán, N., Ávila-Carrera, R., \& Rodríguez-Castellanos, A. (2019). Distribución de fuentes de ruido sísmico y una aplicación a la geofísica. Investigación y Ciencia de la Universidad Autónoma de Aguascalientes, 27(76), 19-31.

\section{RESUMEN}

Mediante la correlación del ruido sísmico es posible recuperar una de las características más importantes de la estructura del medio donde se propagan las ondas sísmicas llamada función de Green. Es necesario estudiar la influencia que guarda la distribución del ruido sísmico para poder estimar si la función de Green recuperada será o no precisa. Este trabajo muestra una formulación que permite obtener la función de Green para diferentes distribuciones de fuentes de ruido sísmico. Primero se utilizan las funciones de Green exactas y se estudian los contenidos de energía de las ondas

Palabras clave: Ondas P y SV; función de Green; distribución de energía; fuentes sísmicas; campos de desplazamiento.

Keywords: P and SV waves; Green's function; energy distributions; seismic sources; displacement fields.

Recibido: 19 de junio de 2018, aceptado: 26 de noviembre de 2018

* Facultad de Ingeniería, División de investigación y Posgrado, Universidad Autónoma de Querétaro. Cerro de las Campanas $\mathbf{s} / \mathrm{n}$, Las Campanas, C. P. 76010, Santiago de Querétaro, Querétaro, México. Correo electrónico: dr.jovanbasalduasanchez@hotmail.com. ORCID: orcid.org/0000-0002-9307-7618

* Centro de Investigación en Matemáticas, A.C. Callejón Jalisco s/n, Mineral de Valenciana, C. P. 36023, Guanajuato, Guanajuato, México. Correo electrónico: nfloresg@gmail.com. ORCID: orcid.org/0000-00015019-6433

*** Instituto Mexicano del Petróleo. Eje Central Lázaro Cárdenas 152 Gustavo A. Madero, C. P. 07730, Ciudad de México, México. Correo electrónico: rcarrer@imp.mx; arcastel@imp.mx. ORCID: orcid.org/00000002-7490-5780

**** Universidad Politécnica del Valle de México. Av Mexiquense s/n, esq, Av. Universidad Politécnica, Villa Esmeralda, C. P. 54910, Estado de México, México. Correo electrónico: arcastel@imp.mx. ORCID: orcid. org/0000-0003-2762-4502

$凶 \quad$ Autor para correspondencia
P y SV. Después, se exhibe la relación de la función de Green exacta y aquella recuperada mediante ruido sísmico. Posteriormente, se analizan varias distribuciones de fuentes sísmicas, principalmente isotrópicas y colineales, y se exponen algunos elementos que permiten valorar cuál de ellas pueden conducir a obtener con buena correlación de la función de Green.

ABSTRACT

By correlating the seismic noise, it is possible to recover one of the most important characteristics of the structure of the medium where seismic waves propagate, such characteristic is known as the Green's function. However, it's necessary to study the influence of the seismic noise distribution in order to estimate whether the recovered Green's function will be accurate. This work shows a formulation that allows obtaining the Green function for different distributions of seismic noise sources. First, the exact Green functions are used, and the energy contents of the P and SV waves are studied. Afterwards, the relationship of the exact Green function and that recovered by seismic noise is shown. Subsequently, several seismic source distributions are analyzed, mainly isotropic and collinear, and some elements that allow us to assess which of them can lead to obtain the Green's function with good approximation are exposed.

INTRODUCCIÓN

Como es bien sabido, al producirse un movimiento sísmico se generan diversos tipos de onda que se propagan a lo largo del medio que las rodea, como es el caso de las ondas de cuerpo, las cuales 
issn 1665-4412, e-issn 2521-9758

Basaldúa-Sánchez, J. E., Flores-

Guzmán, N., Ávila-Carrera, R., \& Rodríguez-Castellanos, A. son divididas en dos grupos: ondas primarias $(P)$ y ondas secundarias (SV). Para el caso de las ondas $P$, presentan un movimiento longitudinal que comprime y dilata el medio en el que se propaga; mientras que las ondas SV presentan un movimiento transversal en comparación con aquellas y no pueden ser propagadas en medios fluidos. Para el análisis de ondas sísmicas, los sismogramas sintéticos son herramientas de gran utilidad en la determinación del tipo de onda que se está propagando, así como la forma de la misma en función de las propiedades físicas del medio con el que interactúan.

Diversos autores se han apoyado en el ruido sísmico para comprender cómo está conformada la tierra, como es el caso de Aki (1957). Adicionalmente, Sato y Fehler (1998) y Aki y Richards (2002) encontraron que información de relevancia referente a las propiedades, estructura y características del medio en estudio, pueden ser encontradas a través del ruido sísmico. Ryzhik, Papanicolau y Keller (1996) señaló que la propagación de energía proveniente del ruido sísmico se consolida a valores constantes que son independientes de la difracción. Posteriormente, Campillo y Paul (2003) y Shapiro y Campillo (2004) concluyeron la factibilidad de recuperar la función de Green utilizando la técnica de correlación cruzada por movimientos sísmicos, dichos autores mostraron gran dependencia en la precisión al momento de recuperar la función de Green del medio en estudio cuando este contiene o no isotropía en las fuentes de excitación sísmicas, además de si es considerado equiparticionado.

Weaver y Lobkis (2004), Wapenaar (2004) y Van Manen, Curtis y Robertsson (2006) en función de medios heterogéneos y homogéneos establecieron identidades relacionadas con la función de Green y la correlación de movimientos sísmicos. Dichos autores enfatizaron que la función de Green que emerge a partir de las correlaciones es completa, simétrica en tiempo e incluye todas las difracciones del medio de propagación. Garnier (2005) propuso una aplicación para construir imágenes en un medio estratificado mediante la correlación cruzada de señales de ruido, enfocando su estudio en la posibilidad de identificar y localizar fuentes a partir de correlaciones cruzadas de señales de ruido.

Stehly, Campillo y Shapiro (2006) mostraron la importancia en la orientación de los receptores en estudios de correlación cruzada para estimar adecuadamente las funciones de Green. Pruebas experimentales actuales orientadas a recobrar la función de Green por medio de ruido sísmico han sido desarrolladas, como es el caso de Garus y Wegler (2011), quienes con una red sismológica europea configuraron una red de 10 estaciones. Los resultados reportados en esta prueba indican que derivado del análisis de ruido sísmico realizado la función de Green no pudo ser reconstruida, a pesar de haber utilizado datos de 17 años de registros.

Por otro lado, Ma y Beroza (2012) pudieron recuperar la función de Green con datos recabados durante 6 meses, mismos que no fueron registrados simultáneamente. Brown y LU (2016) desarrollaron correlaciones aplicadas tanto a mediciones de ondas en tanques como a mediciones de ondas oceánicas, donde los autores reportaron que las simulaciones teóricas coinciden bien en el caso de mediciones en los tanques; sin embargo, para la correlación de ondas oceánicas es necesario considerar los efectos no lineales.

El presente trabajo muestra una formulación que permite estudiar algunos aspectos relacionados con la recuperación de la función de Green mediante correlaciones de ruido sísmico. Primero se hace uso de las funciones de Green exactas y se estudian sus contenidos de energía. Posteriormente se realiza la correlación de ruido y se recupera la función de Green para dos configuraciones de fuentes sísmicas, con discusión de la precisión alcanzada. La formulación se resume en el siguiente apartado.

\section{MATERIALES Y MÉTODOS}

\section{Funciones de Green y ruido sísmico}

Los desplazamientos generados en un medio elástico de volumen $V$ (ver figura 1) pueden expresarse con ayuda de la ecuación de onda elástica en función de la frecuencia como:

$$
\frac{\partial}{\partial x_{j}}\left(c_{i j k l} \frac{\partial u_{l}(x, \omega)}{\partial x_{k}}\right)+\omega^{2} \rho u_{i}(x, \omega)=-f_{i}(x, \omega)
$$

donde $\omega$ es la frecuencia angular, $c_{i j k l}$ es el tensor de constantes elásticas, $p$ es la densidad de masa, $u_{i}(x, \omega)$ el campo armónico de desplazamientos, $f_{i}(x, \omega)$ la distribución de fuerzas de cuerpo 


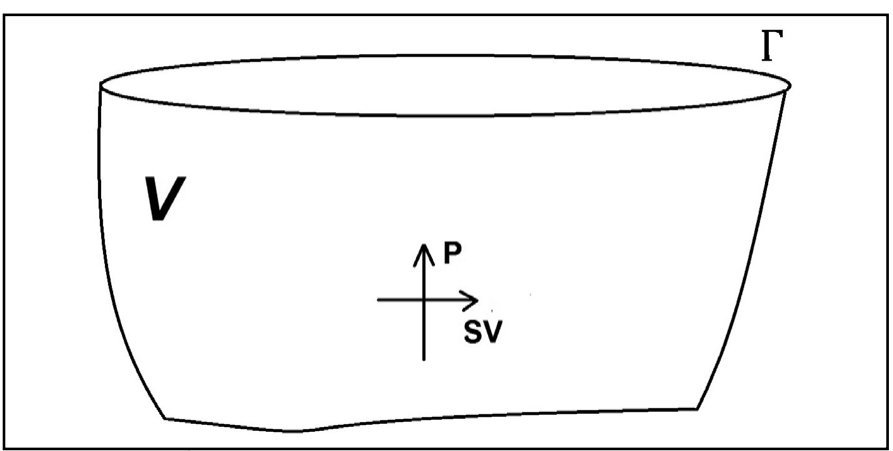

Figura 1. Representación esquemática del medio en estudio, donde $V$ es el volumen del medio, $\Gamma$ la frontera y $P$ \& SV son las fuerzas de cuerpo.

Elaboración propia.

Al aplicar una fuerza unitaria en $x_{A}$, con trayectoria $m$, la fuerza es representada por $f_{i}(x, \omega) \equiv \delta\left(x-x_{A}\right) \delta_{i m} e^{i \omega t}$. El subíndice $m$ especifica la orientación de la fuerza, los desplazamientos generados dependen de la función de Green, teniendo la estructura:

$$
\frac{\partial}{\partial x_{j}}\left(c_{i j k l} \frac{\partial G_{l m}\left(x, x_{A}, \omega\right)}{\partial x_{k}}\right)+\omega^{2} \rho G_{i m}\left(x, x_{A}, \omega\right)=-\delta\left(x-x_{A}\right) \delta_{i m}
$$

El término $G_{i m}\left(x, x_{A}, \omega\right)$ representa la función de Green para desplazamientos; si el término anterior multiplica la ecuación (1), el término $u_{i}(x, \omega)$ la ecuación (2), y se lleva a cabo la diferencia de éstas, se tiene:

$$
\begin{gathered}
\frac{\partial}{\partial x_{j}}\left(c_{i j k l} \frac{\partial G_{l m}\left(x, x_{A}\right)}{\partial x_{k}}\right) u_{i}(x, \omega)-\frac{\partial}{\partial x_{j}}\left(c_{i j k l} \frac{\partial u_{l}(x)}{\partial x_{k}}\right) G_{i m}\left(x, x_{A}\right) \\
=-\delta\left(\left|x-x_{A}\right|\right) u_{m}(x)+f_{i}(x) G_{i m}\left(x, x_{A}\right)
\end{gathered}
$$

Integrando sobre el volumen $V$ restringido por el contorno $\Gamma$, utilizando la formula gaussiana y las relaciones de reciprocidad, la ecuación anterior toma la forma:

$$
\begin{gathered}
\int_{\Gamma}\left\{n_{j}(x)\left(c_{i j k l} \frac{\partial G_{l m}\left(x, x_{A}\right)}{\partial x_{k}}\right) u_{i}(x)-n_{j}(x)\left(c_{i j k l} \frac{\partial u_{l}(x)}{\partial x_{k}}\right) G_{i m}\left(x, x_{A}\right)\right\} d \Gamma_{x} \\
=-u_{m}\left(x_{A}\right)+\int_{V} f_{i}(x) G_{i m}\left(x, x_{A}\right) d V_{x}
\end{gathered}
$$

La frecuencia angular $(\omega)$ ha sido omitida debido a que su dependencia es entendida de aquí en adelante. Dado que $x_{A}$ se ubica dentro del volumen $(V), t_{i}(x)=n_{j}(x)\left(c_{i j k l} \frac{\partial u_{i}(x)}{\partial x_{k}}\right)$ expresa la tracción en el punto $x_{A}$, con normal asociada $n_{j}(x)$, en dirección $i$ en referencia a los desplazamientos $u_{l}(x)$. Además se puede definir $T_{i m}\left(x, x_{A}\right)=n_{j}(x)\left(c_{i j k l} \frac{\partial G_{i m}\left(x, x_{A}\right)}{\partial x_{i}}\right)$ como la tracción en un punto $x$, con normal $n_{j}(x)$, en dirección $i$, concebida por una fuerza unitaria aplicada en el punto $x_{A}$ en dirección $m$, obteniendo así:

$u_{m}=\int_{\Gamma}\left\{G_{i m}\left(x, x_{A}\right) t_{i}(x)-T_{i m}\left(x, x_{A}\right) u_{i}(x)\right\} d \Gamma_{\mathrm{x}}+\int_{V} f_{i}(x) G_{i m}\left(x_{A}, x\right) d V_{x}$

La expresión anterior se conoce comúnmente como identidad de reciprocidad Betti-Rayleigh, la cual relaciona los desplazamientos internos, así como las tracciones y desplazamientos sobre el contorno. Al tener en cuenta que tanto desplazamientos como tracciones son reversibles en el tiempo y al aplicar una fuerza $f_{i}(x) \equiv \delta\left(x-x_{B}\right) \delta_{i n}$ en un punto $x_{B}$ interno en dirección $n$, la ecuación (5) presenta simetría respecto al tiempo y acepta soluciones que también lo son, conduciendo a valores complejos conjugados en el dominio de la frecuencia de la forma $u_{i}(x) \equiv G_{i n}^{*}\left(x, x_{B}\right)$ y $t_{i}(x) \equiv T_{i n}^{*}\left(x, x_{B}\right)$, la ecuación (5) se expresa como:

$$
\int_{\Gamma}\left\{T_{i m}\left(x, x_{A}\right) G_{i n}^{*}\left(x, x_{B}\right)-T_{i n}^{*}\left(x, x_{B}\right) G_{i m}\left(x, x_{A}\right)\right\} d \Gamma_{x}=-G_{m n}^{*}\left(x_{A}, x_{B}\right)
$$

Sustituyendo $x$ por $\xi$ en la ecuación (6), para exhibir los puntos ubicados en el contorno a lo largo de $\Gamma$, la expresión resultante es:

$\operatorname{Im}\left[G_{m m}\left(x_{A}, x_{B}\right)\right]=\frac{-\int_{\Gamma}\left\{G_{m i}\left(x_{A}, \xi\right) T_{i n}^{*}\left(\xi, x_{B}\right)-G_{n i}^{*}\left(x_{B}, \xi\right) T_{i n}\left(\xi, x_{A}\right)\right\} d \Gamma_{\xi}}{2 \mathbb{1}}$

donde Im es la parte imaginaria del tensor de Green. Por otra parte, si se considera que $u_{m}(x)=\int G_{m i}(x, \xi) \phi_{i}(\xi) d \Gamma_{\xi}$ es una representación integral (Sánchez-Sesma \& Campillo, 1991) con densidades de fuerzas $\phi_{i}(\xi)$ a lo largo de $\Gamma$ y se asume que $\phi_{i}(\xi)$ y $\phi_{i}(\xi)$ son recíprocamente no correlacionadas, al considerar además que las funciones de Green de desplazamientos y tracciones son expresadas en términos de funciones asintóticas, es posible escribir la siguiente ecuación:

$$
\left\langle u_{i}\left(x_{A}\right) u_{j}^{*}\left(x_{B}\right)\right\rangle=-4 E_{S} k^{-2} \operatorname{Im}\left[G_{i j}\left(x_{A}, x_{B}\right)\right]
$$

Bajo la configuración 2D, $E_{S}=\rho \omega^{2} S^{2}$ es el promedio de densidad de energía y $S^{2}$ es el promedio de densidad espectral de ondas asociadas a las ondas de corte. $k$ representa el número de onda (para mayor detalle consultar Sánchez-Sesma, Pérez-Ruiz, Luzón, Campillo y Rodríguez-Castellanos (2008)). La ecuación (8) muestra la relación entre la correlación cruzada del campo de desplazamiento 
y la parte imaginaria de la función de Green. Cabe mencionar que esta ecuación implica que la función de Green puede ser recuperada mediante la correlación de movimientos sísmicos; es decir, mediante registros de campo.

Adicionalmente, Sánchez-Sesma et al. (2008) demostraron que en un medio infinito bidimensional se tiene una distribución fija de energía entre dos puntos $\left(\begin{array}{lll}x_{A} & \text { y } & x_{B}\end{array}\right)$ y que dicha energía se distribuye entre los dos tipos de onda que pueden propagarse en el medio (i.e. ondas P y SV). Para el caso de que se tenaan los puntos $A$ y $B$ en la misma ubicación (i.e. $x_{A}=x_{B}$ ): la energía en ese punto puede expresarse como:

$$
\operatorname{Im}\left[G_{i j}\left(x_{A}, x_{A}\right)\right]=-\frac{1}{8 \rho}\left(\frac{1}{\alpha^{2}}+\frac{1}{\beta^{2}}\right) \delta_{i j}=E_{P}^{T}+E_{S V}^{T}
$$

En este punto puede resaltarse que la parte imaginaria del tensor de Green en un mismo punto (fuente-receptor) tiene un valor fijo, puede entenderse que la energía en cualquier dirección será siempre la misma y podrá ser representada como la suma de $E_{P}^{T}$ y $E_{S V}^{T}$ (el superíndice $T$ significa valor teórico). La ecuación (9) sugiere que la distribución teórica de la energía elástica depende solamente de las velocidades de propagación $\alpha$ y $\beta$ (i.e. velocidades de propagación de ondas P y SV, respectivamente). La relación entre las expresiones (8) y (9) es muy valiosa debido a que las correlaciones de movimiento sísmico están relacionadas con la función de Green (ecuación 8) y la función de Green está relacionada con la energía (ecuación 9). Por tanto, es posible expresar el contenido de energía en términos de la correlación del ruido sísmico.

Por otro lado, las contribuciones de energía asociadas a las ondas $P$ y SV pueden extraerse de los sismogramas teóricos y de los sismogramas recuperados resultantes de las correlaciones del ruido sísmico, en términos de la siguiente ecuación:

$$
E^{T}=E_{P}^{T}+E_{S V}^{T} \cong E_{P}^{R}+E_{S V}^{R}=\frac{\rho}{2}\left[\int G_{i j}^{P} d t+\int G_{i j}^{S V} d t\right]^{2}
$$

En la ecuación (10) los superíndices $T$ y $R$ representan la contribución de la energía teórica y recuperada en referencia a las ondas $P$ y SV, respectivamente.

\section{Función de Green exacta}

En un medio isotrópico, elástico y sin fronteras, el tensor de Green exacto en 2D expresa los desplazamientos y tracciones en un punto $x$ cuando una carga unitaria es aplicada en otro punto $\xi$. Asumiendo dependencia temporal armónica $e^{i \omega t}$, donde $i=\sqrt{-1}, \omega$ es la frecuencia angular, y $t$ es el tiempo, el desplazamiento en la dirección $i$ cuando la carga es aplicada en dirección $j$, puede escribirse como:

$$
G_{i j}(x, \xi)=A \delta_{i j}-B\left(2 \gamma_{i} \gamma_{j}-\delta_{i j}\right)
$$

Más aún, la tracción en $x$ en dirección $i$, para cierto vector unitario $n_{i}$ normal a $\Gamma$, producida cuando una carga unitaria es aplicada a $\xi$ en dirección $j$, puede ser escrita como:

$$
\begin{aligned}
& T_{i j}=\frac{\mu}{r}\left\{\left[-4 A B+\lambda \frac{D(\omega r / \alpha)}{2 \mu \alpha^{2}}\right] \gamma_{j} n_{i}+\left[-4 B+\frac{D(\omega r / \beta)}{2 \beta^{2}}\right] \times\left[\gamma_{i} n_{j}+\gamma_{k} n_{k} \delta_{i j}\right]\right\} \\
& +\frac{\mu}{r}\left\{(C+16 B) \gamma_{i} \gamma_{j} \gamma_{k} n_{k}\right\}
\end{aligned}
$$

donde:

$$
\begin{gathered}
A=\frac{1}{i 8 \rho}\left[\frac{H_{0}^{(2)}(\omega r / \alpha)}{\alpha^{2}}+\frac{H_{0}^{(2)}(\omega r / \beta)}{\beta^{2}}\right] \\
B=\frac{1}{i 8 \rho}\left[\frac{H_{2}^{(2)}(\omega r / \alpha)}{\alpha^{2}}+\frac{H_{2}^{(2)}(\omega r / \beta)}{\beta^{2}}\right] \\
C=\frac{D(\omega r / \alpha)}{2 \alpha^{2}}-\frac{D(\omega r / \beta)}{2 \beta^{2}} \\
D(p)=\frac{i}{2 \rho} p H_{1}^{(2)}(p)
\end{gathered}
$$

Donde $\lambda$ y $\mu$ son las constantes de Lamé, $\rho$ es la densidad, $\alpha=\sqrt{(\lambda+2 \mu) / \rho}$ y $\beta=\sqrt{\mu / \rho}$ son las velocidades de onda $\mathrm{P}$ y $S$, respectivamente, $r=$ $\sqrt{\left(x_{1}-\xi_{1}\right)^{2}+\left(x_{3}-\xi_{3}\right)^{2}}, \gamma_{i}=\left(x_{i}-\xi_{i}\right) / r, \delta_{i j}$ es la delta de Kronecker (1 si $i=j, 0$ si $i \neq j$ ) y $H_{m}^{(2)}(\cdot)$ es la función de Hankel de segundo tipo de orden $m$.

RESULTADOS

\section{Distribución de ruido sísmico y recuperación de la función de Green}

Si se considera un espacio 2D caracterizado por 
una relación de Poisson $v=0.25$ (sólido de Poisson) en el cual se localizan cuatro receptores cuyos puntos son: $A=\{-11.314,11.314\}, \quad B=\{11.314,11.314\}$, $C=\{-11.314,-11.314\}$ y $D=\{11.314,-11.314\}$, como se indica en la figura $2 \mathrm{C}$ ), las distancias respectivas entre receptores para $A \& B=22.628 m, B \& D=22.628 m$ y $A \& D=32.000 m$, formando un ángulo de $45^{\circ}$ entre este último par de receptores con respecto al sistema de referencia. De acuerdo con la definición de la función de Green mencionada anteriormente y mediante el uso de las ecuaciones teóricas exactas, es posible propagar ondas elásticas $P$ y SV desde A a B, de $B$ a D y de A a D.

Sus sismogramas sintéticos (exactos) adquieren la forma de las figuras $2 \mathrm{a}, 2 \mathrm{~b}$ y $2 \mathrm{~d}$, cuya nomenclatura $G_{x x}, G_{x z}, G_{z x}$ y $G_{z z}$ se refiere a las componentes del tensor de Green y al sistema mostrado en la figura $2 \mathrm{C}$ ). Para los receptores A \& B (figura 2a)), en el caso de los sismogramas sintéticos de las funciones de Green, puede observarse primeramente el arribo de la onda $\mathrm{P}$ (componente $G_{x x}$ ) y posteriormente el arribo de la onda SV (componente $G_{z z}$ ). Cabe señalar que los valores de las componentes $G_{x z} \& G_{z x}$ son nulas debido a la orientación de los receptores A \& B con respecto al sistema de referencia, se nota además que las ondas SV propagan una mayor cantidad de energía con respecto a las ondas $P$.

Para los receptores $B$ \& D de la figura $2 b$ ), las componentes $G_{x z} \& G_{z x}$ no presentan valor alguno; sin embargo, la componente $G_{z z}$ presenta el arribo de la onda $\mathrm{P}$ y la componente $G_{x x}$ el arribo de la onda SV. Por otro lado, para los receptores A \& D es posible observar componentes de desplazamiento para $G_{x x}, G_{x z}, G_{z x} \& G_{z z}$, (ver figura 2d)), debido a su orientación con respecto al sistema de referencia. En los tres casos expuestos (figuras 2a), b) y d)) se asume que independientemente de la orientación de los receptores la energía que se propaga entre estos debe ser la misma, la cual debe de mantener proporciones fijas para las ondas P y SV. a)

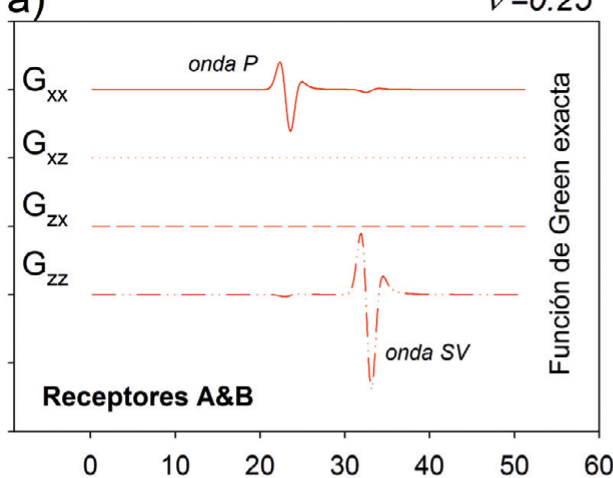

c)

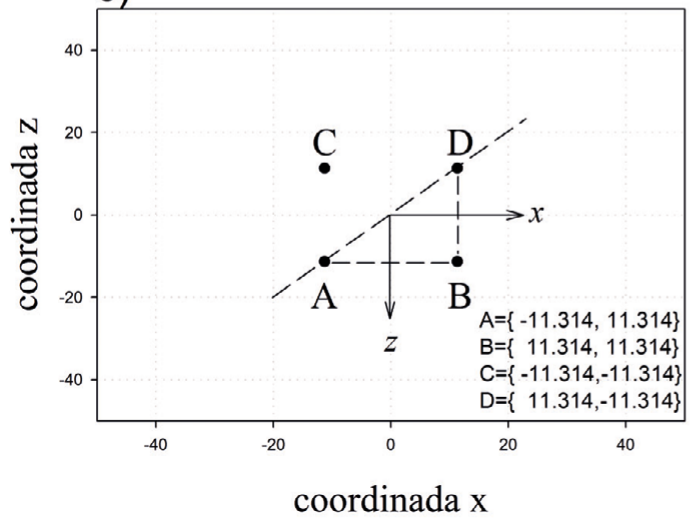

b)

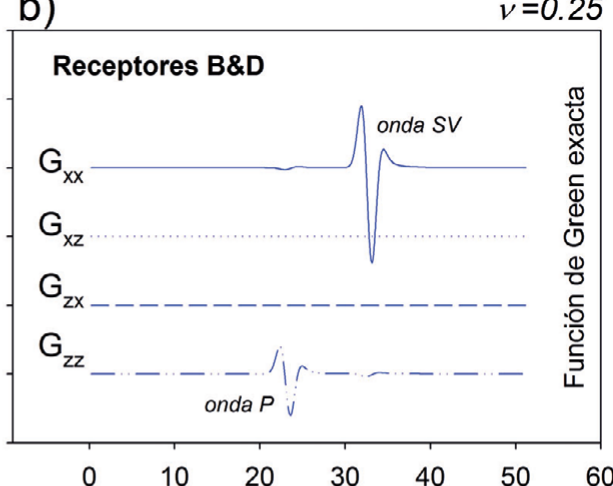

d)

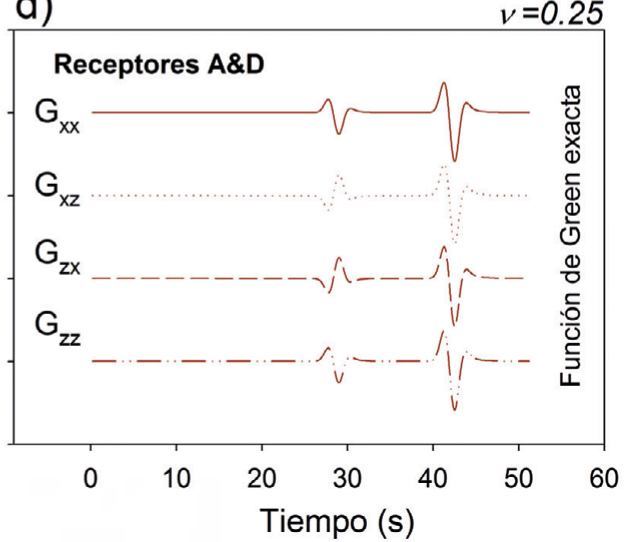

Figura 2. a) Sismogramas sintéticos para receptores A\&B; b) sismogramas sintéticos para receptores B\&D; C) localización de receptores en un espacio con relación de Poisson de $v=0.25, y$ d) sismogramas sintéticos para receptores $A \& D$.

Elaboración propia. 
issn 1665-4412, e-issn 2521-9758

Basaldúa-Sánchez, J. E., Flores-

Guzmán, N., Ávila-Carrera, R., \& Rodríguez-Castellanos, A.

En cualquiera de los casos anteriores (A a B, B a $D$ y $A$ a D) aunque los sismogramas son diferentes la energía propagada entre ellos, en cualquier caso, es la misma sin importar el sistema de referencia. En la ecuación (9) puede verse que la energía que se propaga en un medio depende únicamente de sus propiedades elásticas ( $\alpha$ y $\beta$ ), la cual no tiene relación con el posicionamiento de los receptores en un sistema de referencia. Por otro parte, y con el propósito de mostrar las contribuciones de energía de las ondas P y SV para en un medio elástico, se ha adoptado el criterio de expresar el tipo de medio en función de su relación de Poisson ( $v$ ). La relación de Poisson puede expresarse como el cociente de las velocidades de propagación $\beta$ a $\alpha$ como se muestra en la siguiente expresión:

$$
v=\frac{2\left(\frac{\beta}{\alpha}\right)^{<}-1}{2\left(\frac{\beta}{\alpha}\right)^{2}-2} .
$$

De acuerdo a la ecuación (17) puede concluirse que la energía que se propaga entre un par de receptores es la misma y no depende de la distancia entre ellos o su orientación. De acuerdo con las ecuaciones (9) y (17) se obtiene la figura 3 , en la cual se grafican las contribuciones de energía para las ondas $\mathrm{P}$ y SV con diferentes relaciones de Poisson (v). En esta figura es posible resaltar los valores teóricos (línea continua) obtenidos de la ecuación (9) y los valores obtenidos mediante la ecuación (10) (círculos) de los sismogramas sintéticos, puede observarse buena concordancia entre ambos. Es posible observar también que la mayor aportación de energía corresponde a las ondas SV, mientras que las ondas $\mathrm{P}$ tienen siempre una menor aportación de energía. Para un medio con $v \cong 0$, las ondas SV aportan el $66.66 \%$ de la energía total, mientras que la onda P contribuye con $33.33 \%$ solamente.

Para una relación de Poisson $v=0.25$, se puede afirmar que las ondas SV tienen una contribución de energía de $75 \%$ y las ondas P aportan $25 \%$. Al observar el comportamiento de las curvas de la figura 3 se percibe que las ondas SV aportan una cantidad mayor de energía conforme la relación de Poisson aumenta; por el contrario, las ondas $\mathrm{P}$ aportan menos energía en tales condiciones. Para $v \cong 0.5$, la aportación máxima es dada por la onda SV con $100 \%$ de la energía y sin contribución por parte de la onda P. En la tabla 1 se muestran los valores teóricos de las contribuciones de energía de

las ondas P y SV obtenidas a partir de la ecuación (9) (figura 3, línea continua) versus valores teóricos obtenidos al aplicar la ecuación (10) (figura 3, círculos), en donde se encuentra una gran exactitud con ambas expresiones.

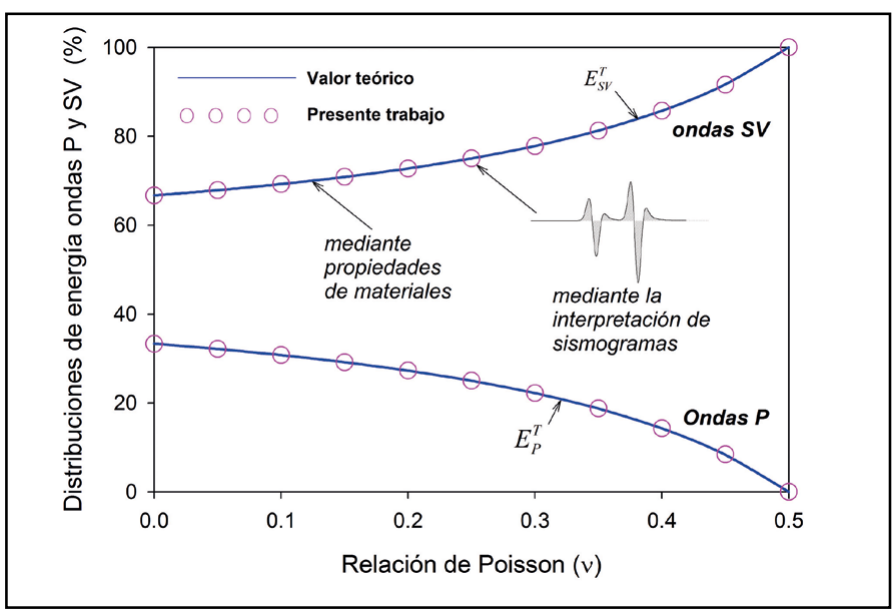

Figura 3. Contribución teórica de las ondas P y SV de la energía total.

Elaboración propia.

Con base en la tabla 1 y mediante las ecuaciones (9) y (10) se pueden obtener las contribuciones de energía exactas de las ondas P y SV para diferentes medios caracterizados por la relación de Poisson. Cabe mencionar que para todos los casos es mayor la contribución de energía de la onda SV con respecto a la onda P. Anteriormente (ver figura 2) se planteó el caso para tres pares de receptores localizados en un medio con una relación de Poisson $v=0.25$ y con un ángulo de referencia para cada par, se mostró que la orientación de los receptores no es un factor que altere la aportación de la energía de las ondas P y SV.

Para los sismogramas sintéticos de la figura 2a) se pudo observar la carencia de energía para las componentes $G_{x z}$ y $G_{z x}$, en cambio, la componente $G_{z z}$ aporta el $75 \%$ de la energía total, mientras que la componente $G_{x x}$ contribuye con $25 \%$. Este resultado puede verificarse en la figura 3 , en donde para un sólido con una relación de Poisson $(v=0.25)$ que se ha venido trabajando, las contribuciones totales de energía mantienen esas cantidades, las ondas P con $25 \%$ y las ondas $S V$ con $75 \%$ de las aportaciones totales de la energía al sistema. Para el caso de la figura 2b), de nuevo se presenta la propagación de ondas $\mathrm{P}$ y SV, solo que ahora la contribución a la energía de las 
Tabla 1

Contribuciones de las ondas P y SV a la energía en función de la relación de Poisson

\begin{tabular}{ccccc}
\hline $\begin{array}{c}\text { Poisson ratio } \\
()\end{array}$ & $\begin{array}{c}\text { Onda P Ecuación } \\
(9)(\%)\end{array}$ & $\begin{array}{c}\text { Onda SV Ecuación } \\
(9)(\%)\end{array}$ & $\begin{array}{c}\text { Onda P } \\
\text { Ecuación (10) (\%) }\end{array}$ & $\begin{array}{c}\text { Onda SV } \\
\text { Ecuación (10) (\%) }\end{array}$ \\
\hline 0 & 33.333 & 66.667 & 33.333 & 66.667 \\
0.05 & 32.143 & 67.857 & 32.143 & 67.857 \\
0.1 & 30.769 & 69.231 & 30.769 & 69.231 \\
0.15 & 29.167 & 70.833 & 29.167 & 70.833 \\
0.2 & 27.273 & 72.727 & 27.273 & 72.727 \\
0.25 & 25.000 & 75.000 & 25.000 & 75.000 \\
0.3 & 22.222 & 77.778 & 22.222 & 77.778 \\
0.35 & 18.750 & 81.250 & 18.750 & 81.250 \\
0.4 & 14.286 & 85.714 & 14.286 & 85.714 \\
0.45 & 8.333 & 91.667 & 8.333 & 91.667 \\
0.499 & 0.002 & 99.998 & 0.002 & 99.998 \\
\hline
\end{tabular}

Nota: Elaboración propia.

ondas SV es aportada por la componente del tensor de Green $G_{x x}$ y para la onda P la energía tiene su presencia en la componente con un $75 \%$ y $25 \%$ del total de la energía, respectivamente.

En la figura 2 d) debido a la orientación de los receptores $\left(\theta=45^{\circ}\right)$ la contribución de la energía corresponde a las cuatro componentes de tensor de Green $\left(G_{x x}, G_{x z}, G_{z x}, G_{z z}\right)$, en donde cada una contribuye con $25 \%$ de la energía. Por tanto, para todos los casos estudiados de la figura 2 las contribuciones de las ondas $\mathrm{P}$ al total de la energía será de $25 \%$, mientras que las ondas SV contribuirán con $75 \%$ para una relación de Poisson $v=0.25$, independientemente de la orientación de los receptores. En la figura 4 se grafican las componentes de las funciones de Green de acuerdo con la orientación de los receptores (ver detalle en figura 4c)l, mostrando para cada caso la aportación total de la energía. Nuevamente se considera un medio con una relación de Poisson $v=0.25$.

Al examinar el ángulo $\theta$ (ver figura $4 c$ )), el cual varia de $0^{\circ}$ a $90^{\circ}$, este determina la orientación de los receptores A y B con respecto al sistema de referencia. Como puede verse en las figuras 4a) y 4b), para las ondas $P$ y SV respectivamente, las contribuciones de energía de cada una de las componentes del tensor de Green para las ondas mencionadas se grafican en función del ángulo $\theta$. Para el caso $\theta=0^{\circ}$ de la onda $P$ (figura 4a)), puede observarse que las contribuciones de energía están dadas por las componentes $G_{x x}$ y $G_{z z}$, y aportan $93.65 \%$ y $6.34 \%$, mientras que la aportación de las componentes $G_{x z} y G_{z x}$ es nula.

Referente a la onda SV se tiene que para $G_{x x}$ la aportación es de $3.46 \%$ y para $G_{z z}$ una contribución de 96.53\% de la energía; del resto de las componentes, es decir, $G_{x z} y G_{z x}$, la aportación tiende a cero. En $\theta=45^{\circ}$, las contribuciones de energía se presentan en todas las componentes del tensor de Green con la misma aportación tanto para las ondas $P$ como para las ondas SV. En la figura $4 a$ ) para las ondas $\mathrm{P}$, las componentes $G_{x x}, G_{x z}, G_{z x}, G_{z z}$ presentan $25.22 \%, 24.77 \%, 24.77 \%$ y $25.22 \%$, respectivamente, de la energía en el sistema, mientras que para las ondas SV, que se puede observar en la figura 4b), la aportación es de $25.10 \%, 24.89 \%, 24.89 \%$ y $25.10 \%$ para cada una de las componentes del tensor de Green. Es notable que las cantidades de energía son equitativas debido a la orientación de los receptores, la energía tiende a repartirse como en la figura $2 d$ ).

En $\theta=90^{\circ}$, la onda $\mathrm{P}$ tiene la mayor contribución y se presenta en la componente $G_{z z}$ con $93.65 \%$, mientras que la energía restante, $6.34 \%$, se presenta en la componente $G_{x x}$. Nuevamente, $G_{x z} y G_{z x}$ tienen contribuciones nulas. Las ondas SV generan un comportamiento similar, obsérvense en conjunto la figura 4b) y la figura 2b), la mayor aportación de la energía, $96.53 \%$, se genera en la componente $G_{x x}$, mientras que para la componente $G_{z z}$ apenas alcanza $3.46 \%$. Al igual que la onda $\mathrm{P}$, 
issn 1665-4412, e-issn 2521-9758

Basaldúa-Sánchez, J. E., Flores-

Guzmán, N., Ávila-Carrera, R., \&

26

las componentes restantes, es decir, $G_{x z} y G_{z x}$, no presentan contribución. En un medio caracterizado por una relación de Poisson $v=0.25$, los gráficos de la figura 4, muestran los valores porcentuales de las contribuciones a la energía de las ondas P y SV obtenidos para cada una de las componentes del tensor de Green para diferentes valores de $\theta$.

La figura 4c) muestra las aportaciones conjuntas de las componentes del tensor de Green a la energía total. Para cualquier ángulo $\theta$ se conservan las contribuciones a la energía del sistema, teniendo que para el caso de la onda P será de $25 \%$ y para la ondaSV de $75 \%$ de la energía (ver figura 3 y figura $4 \mathrm{c}$ )). Cuando $\theta=0^{\circ}$, para la onda $\mathrm{P}$, las aportaciones se hacen por las componentes $G_{x x}$ y $G_{z z}$ teniendo el $23.413 \%$ y $1.586 \%$, respectivamente, como se detalló anteriormente, las componentes restantes no presentan contribuciones a la energía. Para las ondas SV, descartando igualmente las componentes $G_{x z} y G_{z x}$, se tiene que
$G_{x x}$ contribuye con $2.6 \%$, mientras $G_{z z}$ genera $72.399 \%$. Para $\theta=45^{\circ}$, las componentes del tensor de Green para las ondas P aportan $6.307 \%, 6.192 \%, 6.192 \%$ y $6.307 \%$ para $G_{x x}, G_{x z}, G_{z x}, G_{z z}$, respectivamente. Para las ondas SV los valores obtenidos de cada componente corresponden a $18.831 \%, 18.668 \%, 18.668 \%$ y $18.831 \%$ de la energía.

Para $\theta=90^{\circ}, G_{x x} y G_{z z}$ contribuyen con $1.586 \%$ y $23.413 \%$ de energía para el caso de la onda $\mathrm{P}$, como ya ha sido mencionado, el intercambio de energía entre las componentes es notable, puede apreciarse que para las ondas SV ahora la energía mayoritaria tiene presencia en $G_{x x}$ con $72.399 \%$ y $G_{z z}$ con $2.6 \%$ del total de la energía. En todos los casos estudiados, es notorio que la aportación mayoritaria de energía para el medio con relación de Poisson $v=0.25$ viene por las ondas SV con $75 \%$, mientras que para las ondas P la energía tiende a $25 \%$, como puede verse en la figura $4 \mathrm{C}$ ).

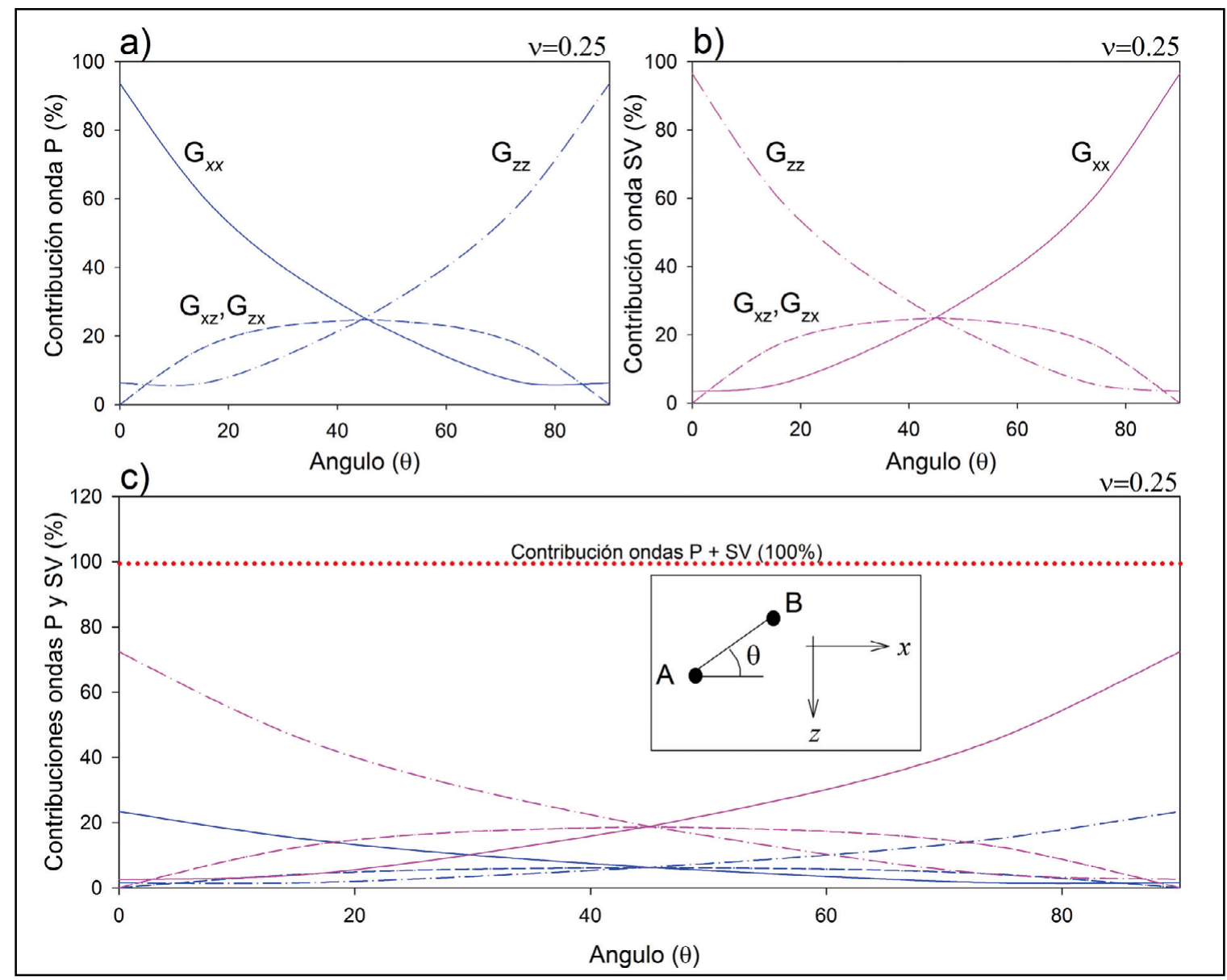

Figura 4. Contribución de energía para cada una de las componentes del tensor de Green en función del ángulo $\theta$. Elaboración propia. 
En la figura 5c) se han dispuesto un par de receptores $A$ \& $B$, cuyas localizaciones vienen dadas por las coordenadas $A=\{-11.314,0\}$ y $B=\{11.34,0\}$ en un medio con $v=0.25$. Para este par de receptores se proponen tres orientaciones de fuentes de ruido (figuras $5 a$ ), $5 b$ ) y $5 d$ )), cuyos valores de $\theta$ son $0^{\circ}, 45^{\circ}$ y $90^{\circ}$, respectivamente. Para este caso se pretende conocer para que ángulo $\theta$ se logra una mejor recuperación de las funciones de Green.
Para la figura 5a) $\operatorname{con} \theta=0^{\circ}$ entre fuentes y receptores, es notable que para las componentes $G_{x x} y G_{z z}$ la función de Green recuperada concuerda con buena aproximación a la exacta. Para este caso, nuevamente las ondas SV contribuyen con $75 \%$ de la energía, mientras que la aportación de las ondas $P$ tienen $25 \%$ de la energía total, las componentes restantes $\left(G_{x z} y G_{z x}\right)$ tienden a cero y su participación a la energía es nula. Las curvas mostradas con línea continua (figura 5a)) denotan

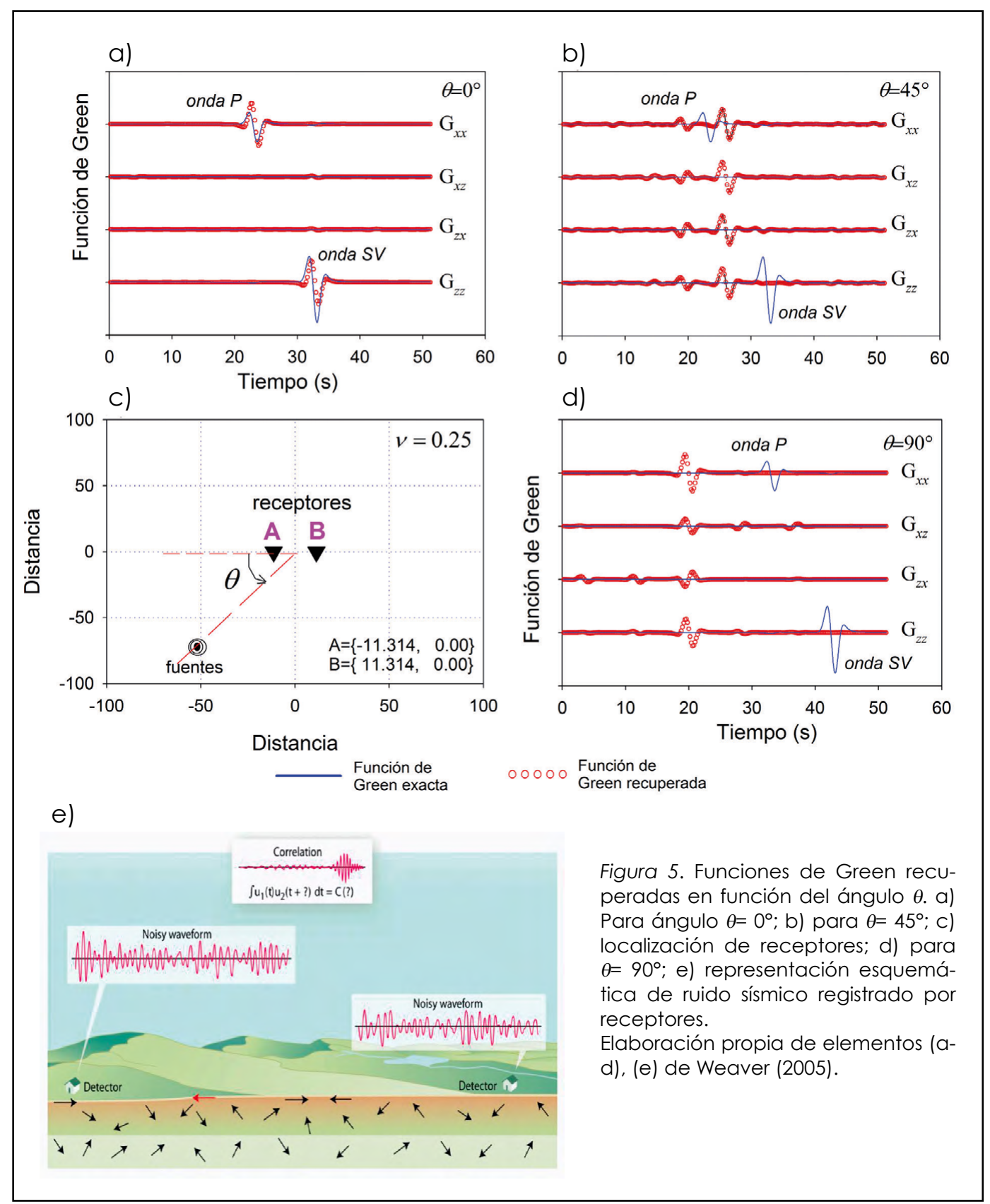


los valores teóricos (exactos), mientras que con círculos se muestran los valores recuperados. Puede verse que la concordancia entre los valores teóricos y recuperados para este ángulo es excelente. En $\theta=45^{\circ}$ (figura $5 \mathrm{~b}$ )) y $\theta=90^{\circ}$ (figura $5 \mathrm{~d}$ )) la recuperación de los valores de Green es muy deficiente.

A partir de la figura 5a) es posible establecer que un sistema de fuerzas orientadas colinealmente con los receptores (A \& B) puede resultar en una recuperación de la función de Green con mayor precisión. Con este mismo fin se muestra que un sistema colineal de fuerzas puede conducir a una estabilización del cociente de energía $E^{R} / E^{T}$, donde la energía recuperada es representada por $E^{R}$ y la teórica por $E^{T}$. Más aún, para que exista una excelente recuperación de la función de Green mediante correlaciones de movimientos sísmicos, el cociente $E^{R} / E^{T}$ debe tender a 1, al mismo tiempo, los valores $E_{P}^{R}$ y $E_{S V}^{R}$ deben tender a sus contrapartes teóricas $E_{P}^{T}$ y $E_{S V}^{T}$. Mediante el uso de la ecuación (10) es posible obtener $E_{P}^{R}$ y $E_{S V}^{R}$ y comparar estos valores con los valores teóricos mostrados en las figuras 3 y 4 .

Para mostrar el comportamiento de $E^{R} / E^{T}$ en función del número de fuentesse han seleccionado tres medios con relaciones de Poisson; $v=0.10,0.25$ y 0.45 . De nueva cuenta, se emplean los receptores y fuerzas colineales según se indica en la figura $6 c$ ). Las figuras 6a), b) y d) corresponden a las relaciones de Poisson; $v=0.10,0.25$ y 0.45 , respectivamente. En estas figuras las líneas sólidas representan la energía recuperada $\left(E^{R}\right)$, los valores teóricos $\left(E^{T}\right)$ son expresados con líneas raya-punto-raya. Las curvas $E^{R}$ muestran un comportamiento similar en las figuras 6a), b) y d) donde puede observarse un fuerte decaimiento desde $N S \cong 100$ a $N S \cong 600$, donde NS es el número de fuentes aleatorias. A partir de este punto, la curva muestra un comportamiento constante hasta alcanzar un NS=10,000.

Para el caso de la figura 6a), se observa que la contribución de $E_{S V}^{R}$ alcanza un valor constante de 0.6923, que representa una contribución de $69.23 \%$ a la energía total. Este valor coincide con el valor teórico esperado $\left(E_{S V}^{T}=69.23 \%\right.$, ver figuras 3 y 4). Para el caso de la energía asociada a las ondas $\mathrm{P}, E_{P}^{R}$ tiende a un valor constante de 0.307, que representa una contribución de $30.7 \%$ al total de la energía (este valor coincide con el valor teórico esperado $E_{P}^{T}=30.77 \%$, ver figuras 3 y 4 ). Para el caso $v=0.25, E_{S V}^{R}$, tiende a 0.75 y $E_{P}^{R}$ tiende a
0.25, los cuales coinciden perfectamente con los valores teóricos mostrados en los gráficos de la figura 4. Similarmente, para $v=0.45, E_{S V}^{R}$ tiende a 0.916 y $E_{P}^{R}$ tiende a 0.083 , los cuales coinciden con los valores teóricos esperados (ver figura 4). Lo anterior da la pauta para interpretar la formulación aquí presentada como correcta.

Es de resaltar que en la figura 6 para NS $<600$ no se obtiene una relación $E^{R} / E^{T}$ aceptable, para NS>600 la relación $E^{R} / E^{T}$ se encuentra muy cercana a 1 y, por tanto, es posible recuperar la función de Green con muy buena precisión. Por otra parte, se concluye que en todos los casos mostrados en esta figura se alcanzan mediante correlaciones de movimiento las contribuciones esperadas para las ondas P y SV, empatando precisamente con el valor teórico.

En este punto se detalla el comportamiento para un par de receptores que están expuestos a dos configuraciones de fuentes sísmicas, fuentes colineales (figura 7a)) y fuentes isotrópicas (figura 7b)). Los receptores están localizados en las mismas coordenadas usadas previamente y el material del medio es representado con $v=0.25$. Cada una de estas fuentes con dirección aleatoria es aplicada en el punto de la fuente y produce desplazamientos en las direcciones $x$ y $z$ en cada receptor (A \& B). Mediante el uso de la ecuación (8) se realiza la correlación de ruido y después se obtiene el sismograma de las funciones de Green recuperadas.

Posteriormente se emplea la ecuación (10) para determinar las contribuciones de energía por cada tipo de onda (P y SV). Para las dos configuraciones de fuentes sísmicas se va incrementando el número de fuentes aleatorias (NS), de tal manera que permite conocer la tendencia del comportamiento de la energía total. Para valores NS desde 100 hasta 10,000 es posible obtener la figura 7c), donde se compara el comportamiento obtenido en función del tipo de sistema de fuente aplicado (colineal o isotrópico). En esta figura la respuesta para el caso isotrópico se grafica con línea punteada, la respuesta para el caso colineal se grafica con línea rayada y los valores teóricos (ver figura 4) se grafican con círculos.

Se observa que el sistema isotrópico requiere un mayor número de fuentes aleatorias para arribar o tender a un valor constante, coincidente con el valor teórico $\left(E_{S V}^{T}=75 \%\right.$ y $E_{P}^{T}=25 \%$, y ver figura 4$)$. Este 


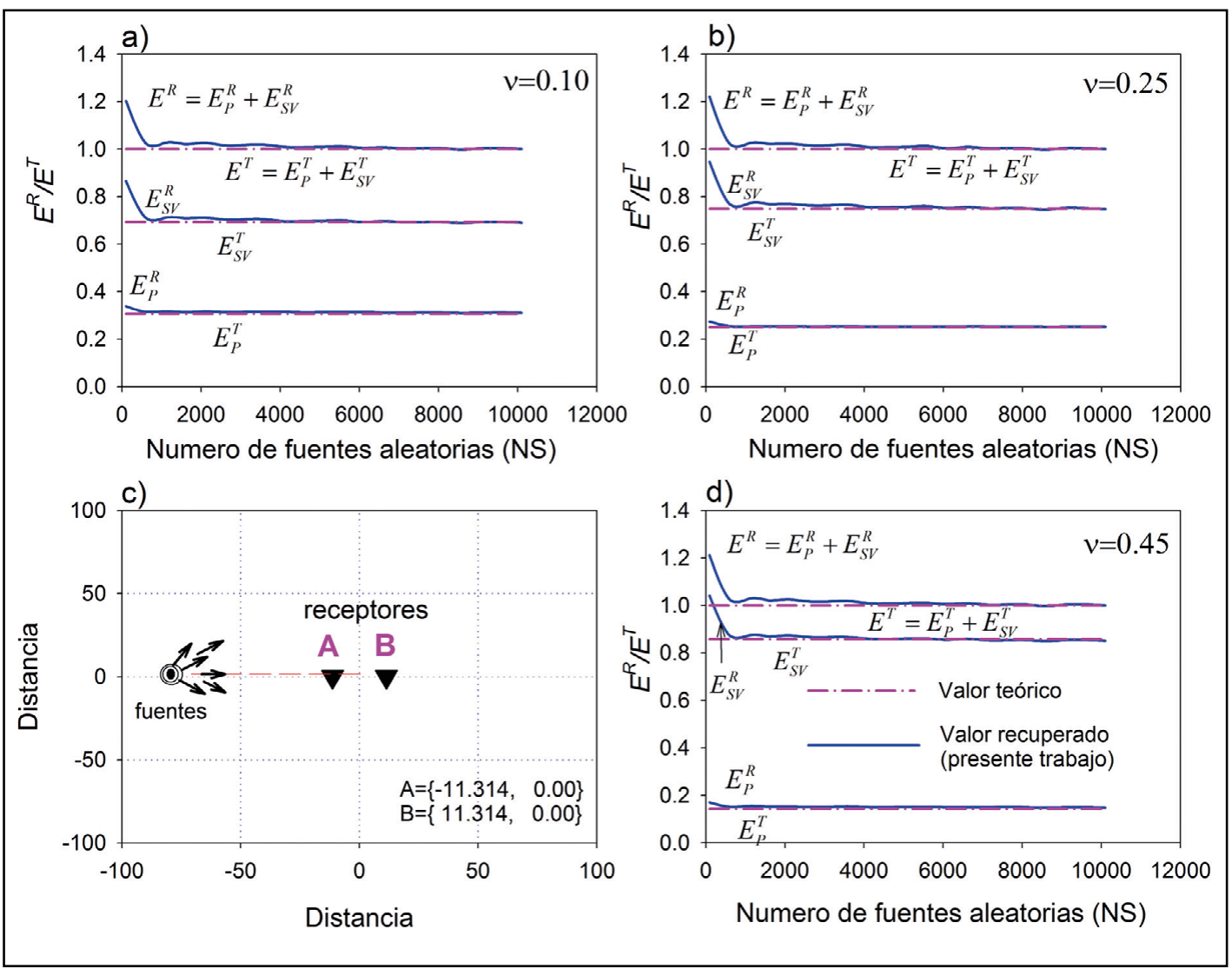

Figura 6. Comportamiento de energía recuperada para un sistema colineal de fuentes sísmicas.

Elaboración propia.

sistema de fuentes isotrópicas requiere de al menos 5,000 fuentes para alcanzar la energía teórica y así poder recuperar la función de Green con buena aproximación. Es decir, no es posible obtener en este caso una buena recuperación de la función de Green mediante correlaciones de ruido si se cuenta con número reducido de fuentes de ruido. Por otra parte, para el sistema de fuentes colineales es posible obtener excelentes contribuciones de energía desde la aplicación de 600 fuentes de ruido, arribando rápidamente a los valores esperados de $E_{S V}^{T} \cong E_{S V}^{R} \cong 75 \%$ y $E_{P}^{T} \cong E_{P}^{R} \cong 25 \%$. En este caso puede concluirse que el sistema colineal de fuentes es más eficiente para recuperar la función de Green con muy buena aproximación.

Obsérvese la línea vertical trazada en el valor de 1000 fuentes aleatorias. Para valores por debajo de esta cantidad, al tenerse una configuración de fuentes sísmicas de ruido isotrópicas, la función de Green no se recupera adecuadamente, mientras al contar con una configuración de fuente colineal la recuperación de la función de Green

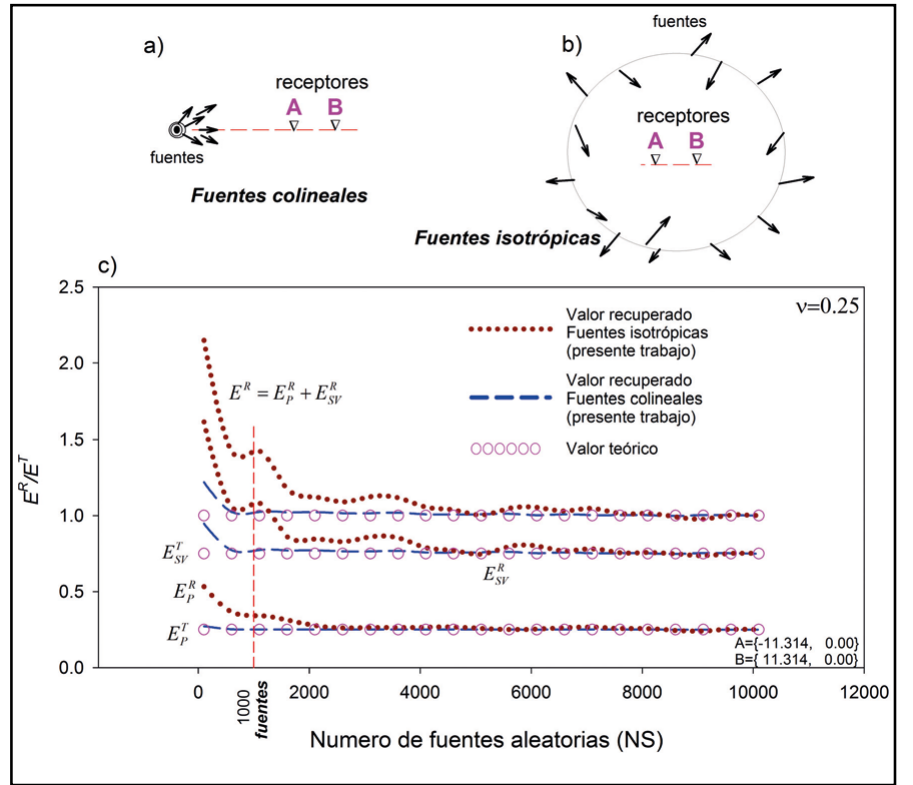

Figura 7. Energía recuperada para dos configuraciones de ruido sísmico. Colineal e isotrópico.

Elaboración propia. 


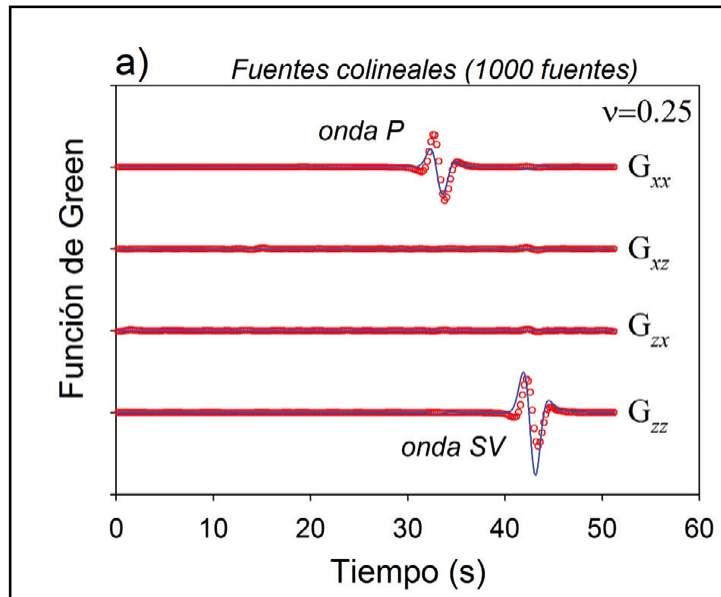

b)

Fuentes isotrópicas (1000 fuentes)

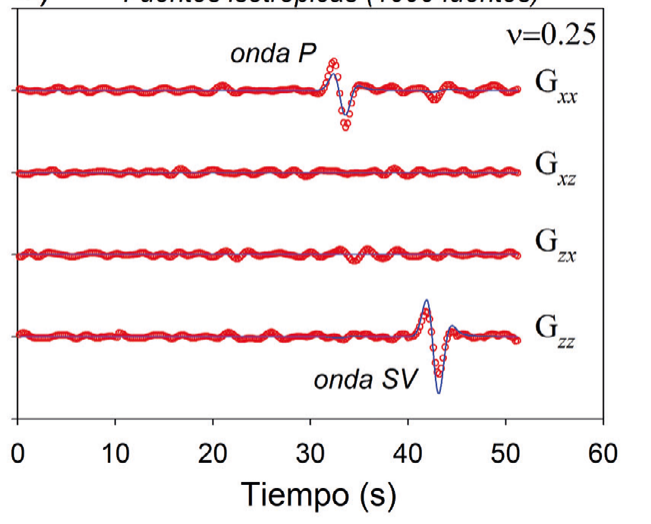

Figura 8. Función de Green recuperadas al aplicar 1000 fuentes aleatorias. a) Sistema colineal del fuentes sísmicas y b) sistema isotrópico de fuentes sísmicas. Elaboración propia.

es sumamente precisa a un número mínimo de 600 aproximadamente. La figura 8 confirma estos resultados para un caso de estudio de 1000 fuentes aleatorias, donde el sistema colineal de fuentes presenta una adecuada recuperación de la función de Green (figura 8a)), la distribución isotrópica de fuentes muestra aún trazas irregulares de la función de Green recuperada comparada con el caso teórico (figura 8b)).

\section{DISCUSIÓN}

Las correlaciones de ruido sísmico han sido usadas recientemente para recuperar una de las características más importantes de los medios donde se propagan las ondas sísmicas (ruido sísmico), la función de Green. En este sentido, es muy importante el desarrollar formulaciones como la mostrada en este trabajo. Como se ha mencionado aquí, la función de Green obtenida recoge todas las reflexiones que se dan en el medio donde se propaga el ruido sísmico $y$, por tanto, contiene las características del medio como sus velocidades y su propia forma y amplitud. Algunos investigadores han puntualizado que para una adecuada recuperación de la función de Green es necesario que exista isotropía en la distribución de las fuentes sísmicas (ver por ejemplo: Campillo \& Paul (2003), Shapiro \& Campillo (2004)).

Sin embargo, en este trabajo se ha encontrado que una distribución colineal de fuentes sísmicas conlleva a una más eficiente recuperación de la función de Green con respecto a la distribución isotrópica. También se encontró que es posible arribar mucho más rápidamente a una función de Green más precisa y con mucha menor cantidad de estas si se aplica un sistema colineal de fuerzas sísmicas. Por otra parte, puede enfatizarse que en todos los casos mostrados en este trabajo fue posible calcular el contenido o distribución de energía por cada tipo de onda sísmica (P y SV) y verificar tal distribución con respecto a los valores teóricos. En este sentido nuestra formulación se muestra como un medio eficiente para el estudio de las correlaciones de ruido sísmico y su relación con la función de Green.

\section{CONCLUSIONES}

Esta investigación muestra una formulación que permite recuperar la función de Green a partir de ruido sísmico. Un aspecto relevante para saber si la recuperación de la función de Green es satisfactoria o no es conocer si la distribución de ruido en el sitio es apropiada y suficiente para recuperar la función adecuadamente. En este contexto se han simulado dos tipos de configuración de ruido sísmico: isotrópico y colineal. También se han estudiado las distribuciones de energía teóricas y aquellas recuperadas mediante ruido, empleando las curvas de distribuciones de energía para observar cuando la recuperación de la función de Green es satisfactoria. Del presente trabajo se pueden enlistar las siguientes conclusiones:

- Una distribución colineal de ruido sísmico conduce a la obtención más precisa de la función recuperada de Green.

- La distribución colineal de ruido sísmico requiere menor número de fuentes sísmicas para obtener 
valores precisos de la función de Green en comparación con la distribución isotrópica.

- Los valores teóricos de energía asociados a las ondas $\mathrm{P}$ y SV poseen valores fijos dependientes de la relación de Poisson del medio, los cuales pueden también obtenerse a partir de la correlación de ruido sísmico.

- La energía tiende a estabilizarse a valores constantes a medida que se incrementa el número de fuentes de ruido sísmico.

- La distribución colineal de fuentes sísmicas es muy conveniente, funciona bien para un rango amplio de relaciones de Poisson $(v=0.10,0.25$ y 0.45$)$.

- Una distribución isotrópica de fuentes requerirá mayor cantidad de ruido sísmico para arribar a valores aceptables de la función recuperada de Green.

\section{Agradecimientos}

Los autores desean agradecer al Instituto Mexicano del Petróleo SENER-CONACYT proyecto (Y.60021) Gas/Oil.

\section{REFERENCIAS}

- Aki, K. (1957). Space and time spectra of stationary stochastic waves, with special reference to microtremors. Bulletin of the Earthquake Research Institute, 35, 415-456. (pp. 11-34). US: University Science Books.

- Brown, M. G., \& Lu, C. (2016). Green's function retrieval in a field of random water waves. Wave Motion, 60, 8-19.

- Campillo, M., \& Paul, A. (2003). Long-range correlations in the diffuse seismic coda. Science, 299(5606), 547-549.

- Garnier, J. (2005). Imaging in randomly layered media by crosscorrelating noisy signals. Multiscale Modeling \& Simulation, $4(2), 610-640$.

- Garus, D., \& Wegler, U. (2011). The Green's functions constructed from 17 years of ambient seismic noise recorded at ten stations of the German Regional Seismic Network. Bulletin of the Seismological Society of America, 101 (6), 28332842.

- Ma, S., \& Beroza, G. C. (2012). Ambient-field Green's functions from asynchronous seismic observations. Geophysical Research Letters, 39(6), L06301. doi: 10.1029/2011GL050755

- Ryzhik, L. V., Papanicolau, G. C., \& Keller, J. B. (1996). Transport equations for elastic and other waves in random media. Wave Motion, 24(4), 327-370.

- Sánchez-Sesma, F. J., \& Campillo, M. (1991). Diffraction of P, SV and Rayleigh waves by topographic features: A boundary integral formulation. Bulletin of the Seismological Society of America, 81 (6), 2234-2253.
- Sánchez-Sesma, F. J., Pérez-Ruiz, J. A., Luzón, F., Campillo, M., \& Rodríguez-Castellanos, A. (2008). Diffuse fields in dynamic elasticity. Wave Motion, 45(5), 641-654.

- Sato, H., \& Fehler, M. C. (1998). Seismic wave propagation and scattering in the heterogeneous Earth. US: Springer-Verlag.

- Shapiro, N. M., \& Campillo, M. (2004). Emergence of broadband Rayleigh waves from correlations of the ambient seismic noise. Geophysical Research Letters, 31 (7), L07614. doi: 10.1029/2004GL019491

- Stehly, L., Campillo, M., \& Shapiro, N. M. (2006). A study of the seismic noise from its long-range correlation properties. Journal of the Geophysical Research, 111, B10306. doi: 10.1029/2005JB004237

- Van Manen, D. J., Curtis, A., \& Robertsson, J. O. (2006). Interferometric modeling of wave propagation in inhomogeneous elastic media using time-reversal and reciprocity. Geophysics, 71 (4), SI47-SI60.

- Wapenaar, K. (2004). Retrieving the elastodynamic Green's function of an arbitrary inhomogeneous medium by cross correlation. Physical Review Letters, 93, 254301-1-254301-4.

- Weaver, R. L. (2005). Geophysics. Information from seismic noise. Science, 307(5715), 1568-1569.

\& Lobkis, O. I. (2004). Diffuse fields in open systems and the emergence of the Green's function. The Journal of the Acoustical Society of America, 116, 2731-2734. doi: $10.1121 / 1.1810232$ 